\title{
Micro discharges in HVDC gas insulated systems
}

\section{Journal Article}

\section{Author(s):}

Schueller, Michael; Gremaud, Robin; Doiron, Charles B.; Franck, Christian (D)

Publication date:

2015-10

Permanent link:

https://doi.org/10.3929/ethz-b-000105301

Rights / license:

In Copyright - Non-Commercial Use Permitted

\section{Originally published in:}

IEEE Transactions on Dielectrics and Electrical Insulation 22(5), https://doi.org/10.1109/TDEI.2015.004835 


\section{(C) 2015 IEEE.}

Personal use of this material is permitted. Permission from IEEE must be obtained for all other uses, in any current or future media, including reprinting/republishing this material for advertising or promotional purposes, creating new collective works, for resale or redistribution to servers or lists, or reuse of any copyrighted component of this work in other works."

Digital Object Identifier: 


\title{
Micro discharges in HVDC gas insulated systems
}

\author{
Michael Schueller ${ }^{1}$, Robin Gremaud ${ }^{2}$, Charles B. Doiron ${ }^{2}$ and Christian M. Franck ${ }^{1}$ \\ ${ }^{1}$ High Voltage Laboratory, ETH Zurich, 8092 Zurich, Switzerland \\ ${ }^{2}$ ABB Switzerland Ltd, Corporate Research, 5405 Baden-Daettwil Switzerland
}

\begin{abstract}
In the present contribution, we analyze and quantify micro-discharge currents originating from rough electrode surfaces in HVDC gas insulated switchgear (GIS) applications. These currents can be significantly larger than those in the gas phase caused by natural ionization or those from bulk conduction in the solid insulators. They may thus dominate the charging of the insulator surface and are thus a key dimensioning criterion.

Five different electrode systems with different, but clearly defined, roughness were designed. To quantify the amplitude of the micro discharge current as a function of the electrical field strength on the electrode surface, the consequence of these discharges in the form of accumulated charges on the surface of a PTFE sample was measured. The measurements were compared to simulation results from a model that uses the Townsend approximation for unipolar saturation ionic current, which is a widely used approximation in many areas of applications. As a main finding of our investigations, to avoid micro discharges, electrical field strengths of $7.1 \mathrm{kV} / \mathrm{mm}$ should not be exceeded in HVDC GIS design using typical industrial electrode roughness around $2 \mu \mathrm{m}$ and 0.5 MPa SF6.
\end{abstract}

Index Terms - SF6, gas insulation, HVDC insulation, charge accumulation, surface charge, ion source, charge carrier, micro discharge, electric conductivity of gases, charge drift in gases.

\section{INTRODUCTION}

HVDC transmission may solve the future problem in power transmission capacity shortage [1]. Due to the increasing integration of renewables, additional transmission capacity is needed in general. Further, long subsea cables, like they are required for offshore wind parks or long sea connections, can only be realized with DC technology as under water compensation is not possible $[1,2]$.

With this expected increasing use of HVDC technology, gas insulated HVDC equipment, such as gas insulated switchgear (GIS) and gas insulated lines (GIL) are expected to become more ubiquitous [1], since their compactness allows important space saving.

Contrary to AC equipment, where the field distribution in operation is determined by the relative electrical permittivity of the different components, the resistivity distribution of the insulation materials governs the stationary electric fields in DC. Consequently, electric field design for gas insulated systems has to be investigated in detail for future DC applications. The main problem in DC is charge accumulation at the boundary layers between different insulation materials. These accumulated charges can influence the electric stress of the insulation considerably. They can reduce the voltage withstand capability and even lead to a breakdown of the insulation system [3]. The most critical parts in $\mathrm{SF}_{6}$ gas insulated DC equipment are the surface of the insulators interfacing $\mathrm{SF}_{6}$ and the solid, and triple points $[4,5]$.

In this article, we investigate the effect of charges arising from the gaseous phase on the solid insulator. There are in principle three different charge sources in the gas: Natural ionization due to cosmic background radiation and radioactive elements in the ground [6], micro discharges from microscopic protrusions (roughness) on the electrodes [6-9], and field emission at highly stressed cathodes or macroscopic protrusions [6-9].

In the literature [10-13] it was shown that in arrangements where partial discharge cannot be detected by classical measurement methods, additional charges due to micro discharge can nevertheless be present. The aim of this contribution is to analyze these micro discharges and quantify the resulting discharge currents and their impact on the charging of HVDC insulator surfaces. For this purpose, an experimental arrangement for testing electrodes with defined roughness grades was designed. The results are compared to simulations.

This paper is organized as follows: In section 2, the theory of electrode roughness and of the generation of micro discharges, as well as the modeling approach are introduced. The test setup and experimental procedure are presented in section 3. Section 4 presents the results from measurement and simulation, which are discussed in section 5 . 


\section{THEORETICAL BACKGROUND AND SIMULATION MODEL}

In case of DC stress, the field distribution is determined by the distribution of the different electric conductivities $\kappa$ of the insulation system. As the direction of the electric field does not oscillate in DC, surface and space charges can accumulate in and on the solid insulation and distort the initial electric field distribution. Charges can be transported in three different ways to the surface of the insulator: by bulk conduction in the solid, by surface conduction or through the gas phase [6-9]. The breakdown voltage reduction due to electrode roughness was studied in detail over the last 40 years. The reduction of the breakdown voltage of arrangements with rough surfaces could be shown as well as the reduction of the PD discharge onset voltage [14-18].

The consequence of partial discharges from protrusions and micro discharges from rough surfaces is a release of additional ions into the gas phase that can accumulate at the insulator surface. In this contribution, only the charges from the gas phase are considered. They originate from natural ionization and micro discharges at the highly stressed roughened electrodes. In this investigation, surface and solid bulk conduction are negligible.

\subsection{GASEOUS DOMAIN AND GENERATION OF CHARGE CARRIERS}

In the gas phase, charges can be produced due to natural ionization, field emission or micro discharges [6-9]. In electro negative gases like SF6, free electrons are quickly attached to neutral gas molecules and it is sufficient to consider only ionic currents.

In the gaseous domain, negative and positive charge carriers (expressed as charge densities $\rho^{-}$and $\rho^{+}$) in an electric potential $\varphi$ move because of the electric field $\boldsymbol{E}=-\boldsymbol{\nabla} \varphi$ and diffusion. The resulting current density can be expressed by the drift-diffusion equation:

$$
\boldsymbol{j}^{ \pm}=\mp \mu^{ \pm} \rho^{ \pm} \cdot \boldsymbol{\nabla} \varphi-D \boldsymbol{\nabla} \rho^{ \pm}
$$

where $\mu$ is the ion mobility and $D$ the diffusion coefficient. At small applied voltages $V$, the current density $j$ increases linearly. For increasing voltages, the current density saturates towards a value that is limited by the ion production rate of natural ionization only as all charges in the gas phase are extracted by the electric field. If the voltage $V$ is increased to a value where the electric field strength in some regions exceeds the critical electric field strength, additional ions are created by impact ionization, which leads to a significant increase in the ion current.

\subsubsection{Natural ionization}

If no partial discharges, micro discharges or field emission are present, natural ionization is the only ion creation mechanism in the gas. This natural ionization is due to cosmic background radiation and radioactive decay events occurring in the vicinity of the gas. Consequently, the ionization rate depends on the exact position of the laboratory where the experiment takes place [19]. In our laboratory $10 \mathrm{~m}$ below ground level, we measured a natural ionization rate $\dot{\rho}_{\mathrm{IP}}=29$ ion pairs $/ \mathrm{cm}^{3} \mathrm{~s}$ in $0.5 \mathrm{MPa}$ SF6.

\subsubsection{Field emission}

Field emission from a highly stressed cathode or protrusions on electrode surfaces can also lead to additional charges in the gas phase. In a healthy insulation system with no protrusions at the electrode, this effect should play a minor role as field strengths in the range of $100 \mathrm{kV} / \mathrm{mm}$ are required for substantial currents to be emitted [8,13].

\subsubsection{Micro discharges}

Local enhancement of the electric field and subsequent impact ionization near rough electrodes (roughness is due to many micro protrusions) on the electrode can create additional charges in the gas phase.

At a given pressure $p$, due to roughness on the electrode surface, the critical field can be exceeded at the micro protrusions (roughness), even when the macroscopic field (neglecting the roughness) does not exceed the critical field strength.

The microscopic shape of every single micro protrusion has an influence on the field enhancement, and hence on the micro discharges. Surface roughness, as it describes the average height of micro protrusions and not their shape, is the most accessible, however not the best parameter for measuring the ability of a surface to induce charges in the gas. For example, two electrodes with the same arithmetic average roughness $R_{a}$, one with slim and sharp micro protrusions will show discharges at much lower field strengths than an electrode with the same measured roughness but broader and rounder shaped micro protrusions [14].

However, a correlation between surface roughness $R_{a}$ and the local field enhancement factor $\beta_{\text {eff }}$ can be observed if the surface roughening process is the same $[14,20]$.

At low field stress, ionization is confined to the high field region near the rough electrode surface, where impact ionization increases locally the density of gas ions. Ions generated near the electrode drift in the gas under the combined influence of the space-charge and external fields.

As the ionization process is very localized and the number of charges generated stays well below the streamer inception criterion, the whole phenomenon is called micro discharge [8]. These small discharges are below the measurement threshold of a classical PD circuit [21] and of any current measurement device.

\subsection{SURFACE POTENTIAL MODELING}

As shown in the previous section, to describe the non-linear nature of the conduction mechanism in the gas, the best approach is to solve the drift-diffusion-reaction equations for the gaseous ions in parallel with the Poisson equation for the electric field [22,23,24]. This is done here with Finite Element Modeling (FEM) numerical simulation (COMSOL) on a rotational symmetric model of the experimental setup described in section 3.2.

\subsubsection{Ion source from natural ionization}

Ion density from natural ionization is represented as a volumic ion source with rate of value $\dot{\rho}_{\mathrm{IP}}=\dot{\rho}^{+}=\dot{\rho}^{-}$. The ionic current from the gas impinging on the PTFE insulator surface is solved according to the model presented in [25]. In practice, this is done by solving the steady-state drift-diffusion equations (1) and (2):

$$
\boldsymbol{\nabla} \cdot \dot{j}^{ \pm}=\dot{\rho}^{ \pm}
$$


for each ion species in the gaseous domain at every time step, making use of the fact that the time needed for newly created ions to drift to a surface is much shorter than all the other relevant timescales in our problem.

\subsubsection{Ion source from micro discharge}

In the particular ring geometry chosen for these experiments, the local field depends on the position on the torus ring surface (macroscopic field $E$ ) and on the shape of the micro protrusion on the rough surface (microscopic field enhancement factor $\beta_{\text {eff }}$ ):

$$
E_{\text {micro }}=\beta_{\text {eff }} E
$$

The additional ionic current induced by the enhanced electric field near the ring electrode is introduced in the model by a boundary condition on the electrode surface.

An empirical expression for the unipolar saturation ionic current was already proposed by Townsend: $I=\mathrm{C}(\mathrm{V}) \mathrm{V}\left(\mathrm{V}-\mathrm{V}_{0}\right)$, with $V_{0}$ being the onset voltage, and $C(V)$ a voltage-dependent prefactor [26, 27, 28]. Due to the self-regulating properties of unipolar space charge drift, the Townsend approximation has a large empirical field of application over many geometrical configuration and operation parameters [29, 30]. From that, the boundary current source for the modeling is adapted from Townsend's relation:

$$
j^{-}=\frac{C(V)}{\Delta z^{2}} \beta_{\mathrm{eff}} E\left(\beta_{\mathrm{eff}} E-p\left(\frac{E}{p}\right)_{\text {crit }}\right)
$$

where $E$ is the macroscopic field at the electrode surface, $\mathrm{p}$ is the pressure, $\left(\frac{E}{p}\right)_{\text {crit }}=88.4 \mathrm{kV} /(\mathrm{mm} \mathrm{MPa})$ is the critical field strength of $\mathrm{SF}_{6}$ [31], and $\Delta z$ is the minimal distance between ring electrode and PTFE sample. Practically, to save simulation time, the macroscopic field $\mathrm{E}$ with semi-ellipsoid shapes is calculated once in a separate run and the ratio of the field from this full simulation to the field with only a smooth half ring is derived. This ratio is applied in the full simulation where only smooth half rings are simulated.

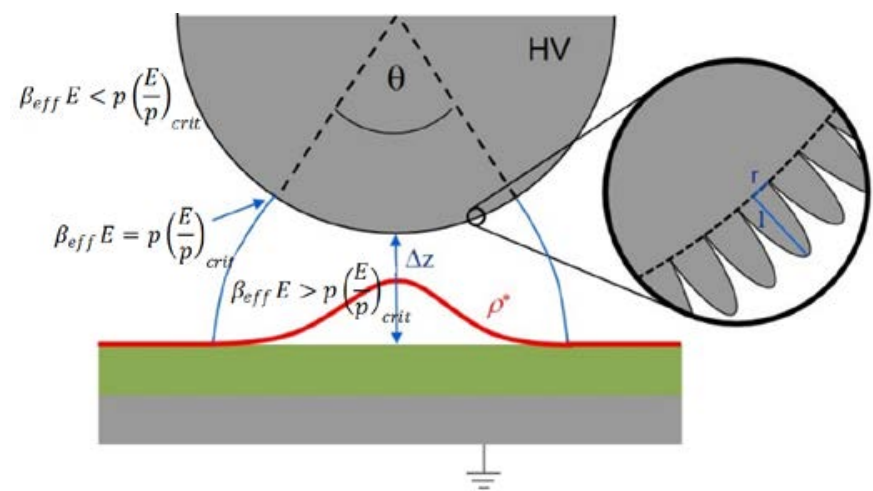

Figure 1. Modeling of roughness and ion source term on the ring electrode surface. Charge emission is non-zero within a circular sector of angle $\theta$. The semi-ellipsoid shaped micro protrusions are not simulated directly; their presence is modelled via the $\beta_{\text {eff }}$ factor in equation (4).

Contrary to corona-wire type experiments [32], in our experiments the macroscopic field on the $\mathrm{HV}$ electrode is nowhere close to the critical field on the electrode surface. Consequently, in the modeling, the ion source term should depend on the location on the electrode and on the level of surface roughness: The roughness is represented by an array of semi-ellipsoids on the ring electrode surface (see Figure 1). The ratio $l / r$ between the axes of the ellipsoid defines the effective field enhancement factor at the tip of the micro protrusion $\beta_{\text {eff }}$ [33]. Note that $\beta_{\text {eff }}$ only contributes to the electrode boundary condition in equation (4), the electric field within the finite-element model being calculated from the macroscopic radius of the ring (dashed line in zoom of Figure 1). For a given applied voltage $V, \beta_{\text {eff }}$ determines the angle $\theta$ where micro-discharges occur as long as the condition $\beta_{\mathrm{eff}} E>p\left(\frac{E}{p}\right)_{\text {crit }}$ is met. If the voltage is increased, the macroscopic electric field and the angle $\theta$ increase as well. Moreover, the microscopic field in front of blunter micro protrusions now overcomes the breakdown condition. As a consequence, micro discharges also start at these blunter micro protrusions: this is reflected by an increase of the voltagedependent prefactor $C(V)$.

\subsubsection{Surface charge and surface potential}

In this paper, to analyze and quantify the micro discharge current at the electrode, the consequence of the discharges in form of electric potential caused by accumulated charges on the surface of the PTFE sample is measured instead. Since PTFE has a high bulk and surface resistivity, conduction currents from the bulk and along the surface are negligible. Therefore, only the gas phase charging of the PTFE surface needs to be considered in the present experiments, and is introduced as a boundary current source on the gas-solid interface:

$$
n_{g} \cdot j_{g}=\partial_{t} \rho^{*}
$$

where $n_{g}$ is the normal vector pointing from the surface of the PTFE sample in the gas, $j_{g}=j^{+}-j^{-}$the current density from the gas phase and $\rho^{*}$ the surface charge. So-called "open" boundary conditions are applied to the other interfaces ( electrodes and grounded tank), i.e. using $j^{ \pm}=\mp \mu^{ \pm} \rho^{ \pm} \cdot \nabla \varphi$ in regions where the charges flow out of the domain, and $\boldsymbol{j}^{ \pm}=0$ otherwise.

Electric field and surface potential are then calculated using the Poisson equation:

$$
\rho^{*}=\boldsymbol{\nabla} \cdot\left[\varepsilon_{0} \varepsilon_{r}(-\nabla \varphi)\right]
$$

\section{Methods}

\subsection{TEST VESSEL AND MEASUREMENT SYSTEM}

To investigate the charging process of GIS insulators in $\mathrm{SF}_{6}$ under DC stress, a gas insulated setup was designed and constructed that combines the flexible exchange of electrodes and insulator samples with an accurate, reliable, and automated surface potential measurement. The test setup was described in detail in [10,34]. 


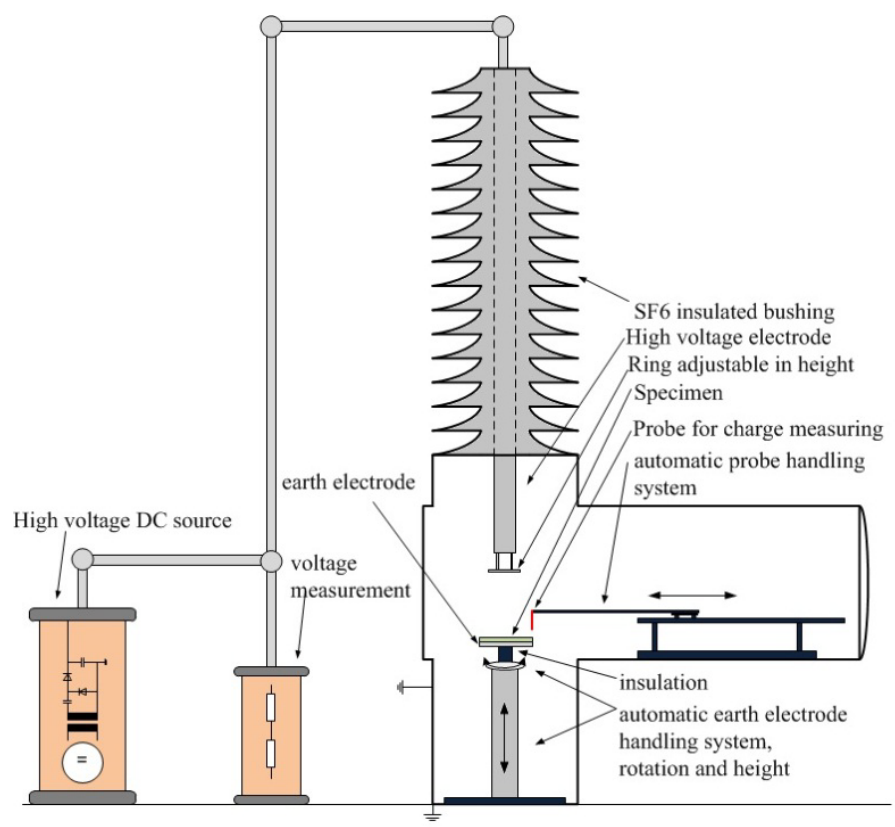

Figure 2 Schematic view of the test set up with high voltage supply and measurement on the left, and the test specimen and surface potential measurement system on the right. The sketch shows the position of the sample during measurement.

During the charging of the sample, the measurement sensor is in its parking position away from the test sample surface on the right-hand side in Figure 2. To measure the surface potential, the high voltage supply is first switched off and the PTFE plate is moved down to a level where the sensor can scan the surface. Then the sensor on the horizontal axis is moved towards the sample. By moving the probe and rotational motor systems, the surface of the sample is passed along the sensor head. The measured voltage signals are correlated with the positions of the handling system to map the measured surface potential.

A DC-Stable Electrostatic Voltmeter 347 from Trek was used as measurement device. This is a high precision electrostatic voltmeter using the principle of a vibrating Kelvin probe. The sensor is mounted on the horizontal axis of the automatic handling system and is positioned with $3 \mathrm{~mm}$ distance to the surface of the test sample when measuring [34].

The Kelvin probe has a sensitivity level of $1 \mathrm{~V}$ and a step of $1 \mathrm{kV}$ can be followed within $3 \mathrm{~ms}$. Fast measurement procedures are possible and the impact of the measurements on the surface charge distribution is small [34]. A resolution of $5 \mathrm{~mm}^{2}$ can be achieved placing the sensor $3 \mathrm{~mm}$ away from the sample surface. The overall measurement repeat accuracy of the setup with another electrode configuration is 10\% of the measured value [34]. The probe was calibrated before every measurement by setting it exactly to zero when measuring the potential of the grounded frame on the sample upper side.

\subsection{TEST SPECIMEN \& ELECTRODE ARRANGEMENT}

The aim of this contribution is to analyze and quantify micro discharges occurring at electrodes due to surface roughness. The test setup is thus designed to fulfill the following requirements: a) The surface of the electrode should have a defined roughness.

b) Changing the roughness of the electrode should be possible in an easy way.

c) The sample material should have a high bulk and surface resistivity.

d) The electric field lines connecting the surface of the sample with the rough electrode should only meet a certain defined part of the sample surface.

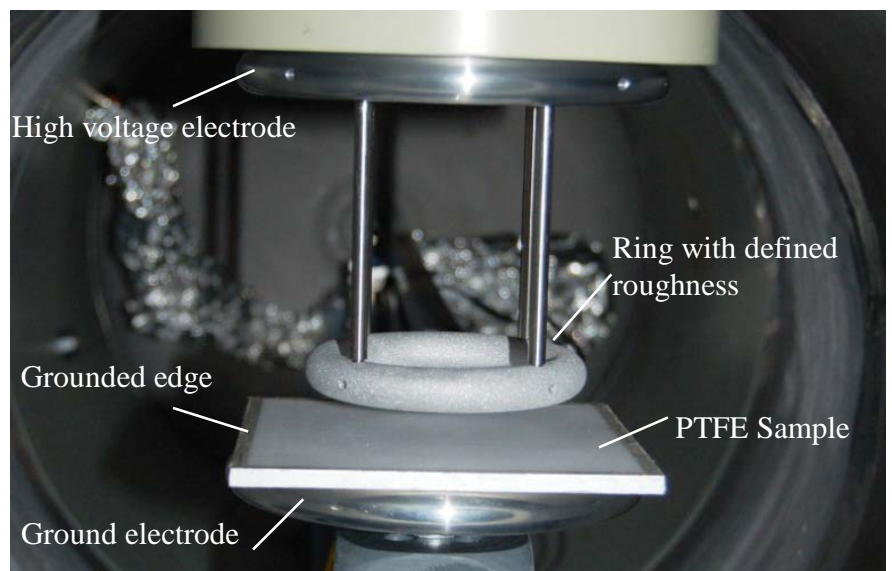

Figure 3 Photo of the electrodes and sample configuration. Note that during charging, the spacing between the electrode and the sample is smaller than depicted here.

The sample and electrode geometry that was chosen and designed is shown and plotted in Figure 3 and Figure 4. The test samples are made of PTFE, because it has a high bulk resistivity of $>10^{20} \Omega \mathrm{m}$ and surface resistivity of $>10^{18} \Omega$ square [35]. The permittivity of the PTFE used is $\varepsilon_{\mathrm{r}}=$ 2.1. The samples are square plates with $140 \mathrm{~mm}$ side length and $5 \mathrm{~mm}$ thickness. The bottom side of the sample was treated with conductive silver paint to ensure no potential difference between the grounded electrode and the sample. Further, the ground potential was brought with this conductive paint over the side of the sample up to a $5 \mathrm{~mm}$ frame around the sample on the upper side. This was done to make a calibration of the sensor possible and to reduce the impact of the edges of the sample. An active area of $130 \times 130 \mathrm{~mm}^{2}$ was conserved on the upper side.

The active part of the high voltage electrode system consists in a ring shaped electrode. The inner diameter of the ring is $70 \mathrm{~mm}$ and the outer diameter $100 \mathrm{~mm}$. The rings can be easily exchanged at the mounting system. Considering a cut through view of the experiment, the centers of the metallic part of the rings would be located at position $-42.5 \mathrm{~mm}$ and $42.5 \mathrm{~mm}$ on the active surface of the sample.

Three different types of rings with defined roughness were produced by sandblasting. To have a perfectly smooth ring surface with nearly no imperfections and field enhancement, one ring was polished. The resulting RMS roughness is $R_{a}=(0.06 \pm 0.005) \mu \mathrm{m}$. The other rings were sandblasted with grain size F80 and F1000 resulting in a surface roughness of $R_{a}=(2.1 \pm 0.18) \mu \mathrm{m}$ and $R_{a}=(10.9 \pm 2.5) \mu m$. Further one set of rings was coated after the sandblasting procedure with $60 \mu \mathrm{m}$ epoxy-based, metal-oxide filled dielectric paint of $10^{-11}$ $\mathrm{S} / \mathrm{m}$ DC conductivity. The RMS roughness of the roughest 
rings (F1000) after coating was $R_{a}=(0.61 \pm 0.08) \mu m$ with a few single sharp metal tips still sticking out of the paint.

The PTFE plate sample is placed at a distance $\Delta z=5 \mathrm{~mm}$ from the high voltage ring electrode. This leads to electric field strengths within which micro discharges should occur. As the gap is quite small it has to be ensured that the rings and the sample were placed perfectly horizontal into the set up. If, for example, the sample is put a little aslope on the earth electrode, the electric field strength would be much higher on one side of the sample because the gap is a little smaller there. To avoid this effect, the sample and rings were installed with great care with a highly precise mechanic's level to guarantee proper alignment.

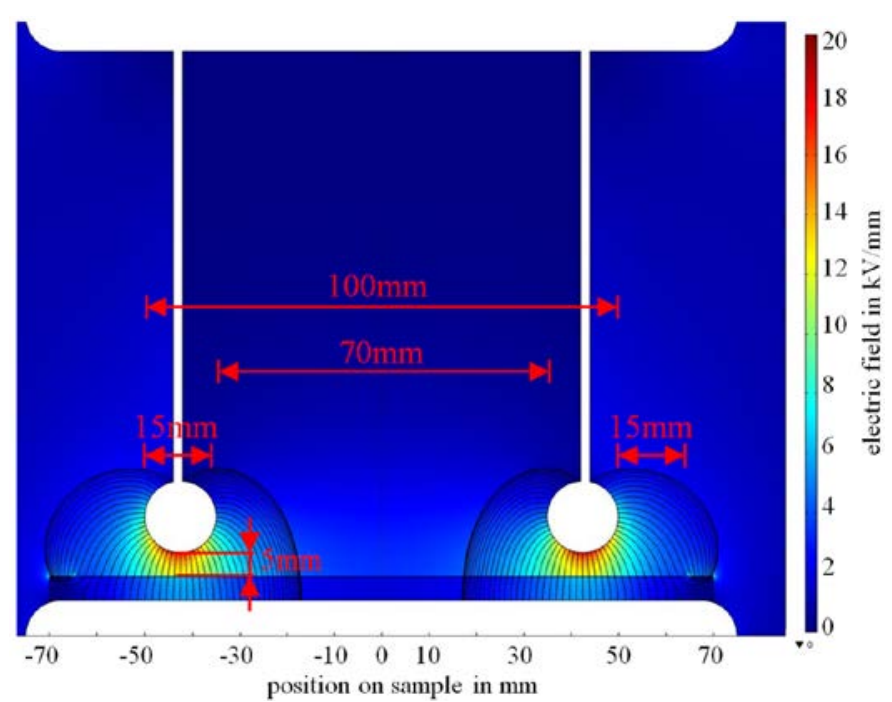

Figure 4 Field calculation for $100 \mathrm{kV}$ of the test arrangement with high voltage electrode (top), ring with field lines, ground electrode (bottom), and test specimen and electric field lines.

The highest electric field strengths are reached at the bottom of the rings. With a voltage of $100 \mathrm{kV}$, the maximal macroscopic electric field strength is $17.7 \mathrm{kV} / \mathrm{mm}$. This is well above the maximal electric field strength used for designing GIS AC equipment and well below the critical field strength of $\mathrm{SF}_{6}$ at $0.5 \mathrm{MPa}$.

Furthermore, in $0.5 \mathrm{MPa} \mathrm{SF}_{6}$ gas this value is clearly lower than the measurable $\mathrm{PD}$ onset voltage. A partial discharge measurement according to IEC 60270 standard was performed. The entire setup showed a based level of $4.4 \mathrm{pC}$ at $100 \mathrm{kV}$. Using the roughest ring F1000 with a roughness of $10.9 \mu \mathrm{m}$, no partial discharges could be detected up to a voltage of $100 \mathrm{kV}$ DC. As no PD onset could be measured with the roughest ring, no more partial discharge measurements were performed with the other, smoother rings.

The active gas volume $[9,36]$ is the volume that is connected via field lines to the surface of the insulator. Only charges inside the active gas volume will drift towards the insulator surface and accumulate. In the present set up, the active gas volume is around $1300 \mathrm{~cm}^{3}$. With the ion production rate $\dot{\rho}_{\text {IP }}=29$ ion pairs $/ \mathrm{cm}^{3} \mathrm{~s}, 1.38 \cdot 10^{8}$ ion pairs were produced after one hour in the active gas volume by natural ionization. That corresponds to a charge $\mathrm{Q}=2.22 \cdot 10^{-11} \mathrm{C}$. In the present electrode configuration this leads to a surface potential in the order of $1 \mathrm{~V}$. In other words, if the measured surface potential increases by a few $\mathrm{V} / \mathrm{h}$ only, natural ionization is the responsible charge source.

\subsection{EXPERIMENTAL PROCEDURE}

The micro discharges are investigated by a step up experiment procedure with voltage steps of $5 \mathrm{kV}$. The maximal applied voltage is $100 \mathrm{kV}$ DC negative polarity. The voltage level is applied for one hour and after that time the surface potential is measured and compared to the surface potential measured at the step before. Due to measurement limitations, a maximal surface potential of $3 \mathrm{kV}$ can be measured. If only a slight increase of the surface potential is detected, the voltage is increased once more. The increase of the micro discharge current can be easily seen with this procedure as the measured surface potential increases strongly in a short period of time.

Particular care has to be taken when installing the insulator sample, as the effect of a slightly misaligned sample is significant. The gap between the ring and the PTFE sample is only $5 \mathrm{~mm}$. If the sample is lying just a little aslope on the earth electrode, then the field strength is changed. If the sample is $0.5 \mathrm{~mm}$ aslope resulting in a gap of $5.5 \mathrm{~mm}$ on one side and $4.5 \mathrm{~mm}$ on the other side, the maximal field strength is decreased by $5.1 \%$ and increased by $6.2 \%$, respectively. This has a noticeable impact on the current amplitude from the micro discharges. An increase of voltage of $5 \mathrm{kV}$, like it was done in the step up experiments, increases the electric field strength by $\sim 7 \%$ as well. This effect can be seen in Figure 5. Further, it has to be mentioned that in case of a slightly misaligned sample also the measuring gap between sensor and sample is not always $3 \mathrm{~mm}$. The influence of spacing between sample and sensor was investigated in [34] in detail. According to these findings, this effect has small impact on our present measurements.

\section{Results}

All measurements and simulations were done with an $\mathrm{SF}_{6}$ pressure of $0.5 \mathrm{MPa}$ absolute with the electrode and sample configuration as shown in Figure 3 and Figure 4.

The increase of surface charges is measured after every hour. By measuring $\Delta \mathrm{V}$ after one hour, it is possible to determine the increase of micro discharge currents in the gas phase.

\subsection{LOW SURFACE ROUGHNESS}

Surface potential measurement on the PTFE plate were done after $1 \mathrm{~h}$ voltage application of sequentially setting $-85 \mathrm{kV}$, $90 \mathrm{kV},-95 \mathrm{kV}$ and $-100 \mathrm{kV}$ DC on the polished ring. The initial surface potential increased uniformly at most by $3 \mathrm{~V}$ per voltage step and hour, and no clear signature of the position of the ring could be found.

\subsection{MEDIUM SURFACE ROUGHNESS}

In Figure $5 \mathrm{a}$ ), the result of a measurement with the ring sandblasted with F80 grain size $\left(\mathrm{R}_{\mathrm{a}}=2.1 \mu \mathrm{m}\right)$ is shown.

Applying $-60 \mathrm{kV}$ (resulting in a macroscopic field strength at the ring surface of $10.5 \mathrm{kV} / \mathrm{mm}$ ) to the arrangement for 3 hours did not result in a surface potential higher than $15 \mathrm{~V}$ (magenta line). Increasing the voltage to $-65 \mathrm{kV}$ $(11.5 \mathrm{kV} / \mathrm{mm})$, some additional charges could be observed 
around position $\mathrm{y}=40 \mathrm{~mm}$, resulting in a maximal surface potential of $-150 \mathrm{~V}$ after one hour and $-180 \mathrm{~V}$ after 3 hours (green and cyan lines). At the other ring position around position $\mathrm{y}=-40 \mathrm{~mm}$, no additional discharges could be observed.

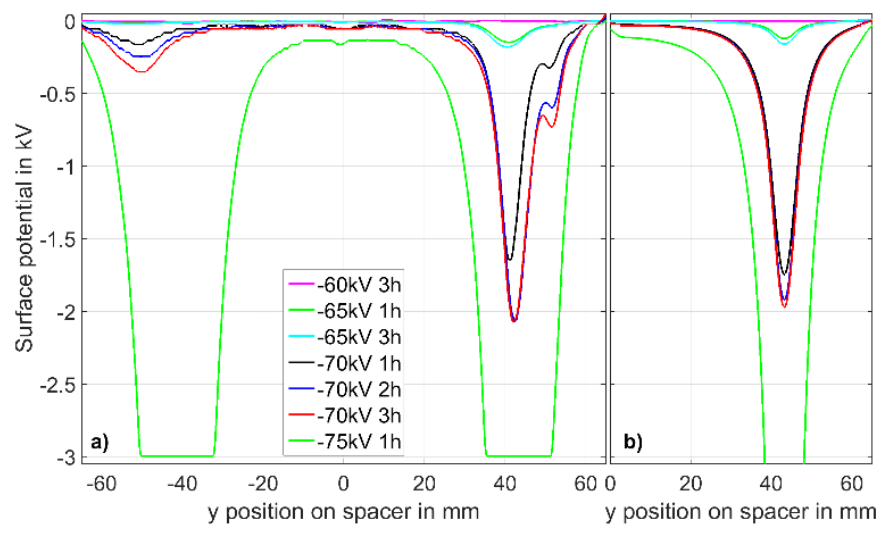

Figure 5 Surface Potential on the PTFE sample as a function of applied voltage and time for the $2.1 \mu \mathrm{m} \mathrm{R} \mathrm{R}_{\mathrm{a}}$ electrode. a) Measurement, b) Modeling.

Increasing the voltage to $-70 \mathrm{kV}(12.4 \mathrm{kV} / \mathrm{mm})$ results in additional charges producing a peak with $-1650 \mathrm{~V},-2050 \mathrm{~V}$ after 1,2 and 3 hours at position $\mathrm{y}=40 \mathrm{~mm}$ and a small surface potential increase on the other side of the sample $(y=-$ $45 \mathrm{~mm}$ ) where the other position of the ring was (black, blue and red lines). In addition, a very local increase of the potential at position $\mathrm{y}=52 \mathrm{~mm}$ can be observed (outer side of ring).

Applying $-75 \mathrm{kV} \quad(13.3 \mathrm{kV} / \mathrm{mm})$ for one hour to the arrangement results in a very high surface potential at both sides of the sample exceeding the measurement range of the probe (green line). The position of the peaks of the surface potential reflects the geometrical position of the ring in this cut-through measurement.

In these experiments, the sample was most probably not perfectly aligned. That led to a higher field strength around position $\mathrm{y}=40 \mathrm{~mm}$ as the sample was a little closer to the electrode on this side. Consequently, on this side of the sample additional discharges start at a lower voltage level than on the other side, where the field strength was lower.

Figure $5 \mathrm{~b}$ ) shows the simulated surface potential curves for the F80 ring at $5 \mathrm{~mm}$ spacing between sample and ring. The modeled surface potential includes the convolution with the spatial response function of the measurement sensor. Field enhancement factors used are $\beta_{\mathrm{eff}}=3.8$ for measurements at $60 \mathrm{kV}$ and $-65 \mathrm{kV}$ and $\beta_{\text {eff }}=3.6$ for voltages below. Semiellipsoids of aspect ratios $\mathrm{l} / \mathrm{r}=1.2-1.3$ are assumed. With these parameters, it is possible to reproduce the saturation in surface potential after 3h (Figure 6).

The prefactor $C(V)$ increases for each voltage step up to $-70 \mathrm{kV}$ absolute voltage, indicating that an increasing fraction of the rough surface contributes to micro-discharges between voltages $(-60 \ldots-70 \mathrm{kV})$.

\begin{tabular}{|l|l|l|l|}
\multicolumn{3}{c}{ F80 Ring } & \multicolumn{2}{c|}{ F1000 Ring } \\
Voltage [kV] & $\boldsymbol{C}(\mathbf{V})\left[\mathrm{Am}^{2} / \mathbf{V}^{2}\right]$ & Voltage $[\mathbf{k V}]$ & $\mathbf{C}(\mathbf{V})\left[\mathrm{Am}^{2} / \mathbf{V}^{2}\right]$ \\
\hline $\mathbf{- 6 0}$ & 0 & -20 & $1.5 \times 10^{-31}$ \\
\hline $\mathbf{- 6 5}$ & $4.3 \times 10^{-27}$ & -30 & $2.3 \times 10^{-31}$ \\
\hline $\mathbf{- 7 0}$ & $1.5 \times 10^{-26}$ & -40 & $3.3 \times 10^{-31}$ \\
\hline $\mathbf{- 7 5}$ & $1.5 \times 10^{-26}$ & -50 & $6.6 \times 10^{-31}$ \\
\hline
\end{tabular}

\begin{tabular}{|l|l|l|l|}
\hline & & -55 & $8.0 \times 10^{-31}$ \\
\hline & & -60 & $9.3 \times 10^{-31}$ \\
\hline & & -65 & $9.3 \times 10^{-31}$ \\
\hline
\end{tabular}

Table 1 Voltage-dependence of the prefactor $C(V)$.

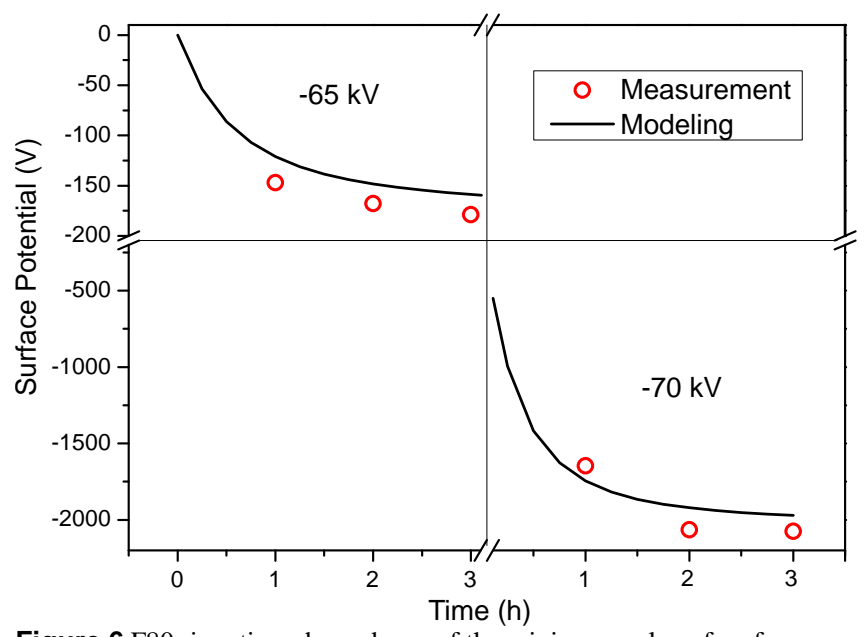

Figure 6 F80 ring, time-dependence of the minimum value of surface potential.

The width of the profile is, however, underestimated (see $-75 \mathrm{kV}$ curve). This implies that the Townsend approximation is a too steep current-voltage characteristic in this case.

\subsection{HIGH SURFACE ROUGHNESS}

In Figure 7, the results of measurements and simulations of the ring sandblasted with F1000 grain size $\left(\mathrm{R}_{\mathrm{a}}=10.9 \mu \mathrm{m}\right)$ are shown.

The position of the ring can be clearly seen as there are peaks observed in the surface potential. In these experiments with the roughest ring, micro discharges can be observed already with lower applied voltages. Even the application of only $20 \mathrm{kV}$ resulted in a measured surface potential of $50 \mathrm{~V}$ after 3 hours at the location of the rings, but not at other locations (green curve). Thus, already at this lowest applied voltage first indications of micro-discharges can be observed. After three hours of applying $-30 \mathrm{kV}$, the surface potential increased to $135 \mathrm{~V}$ at location $\mathrm{y}=-35 \mathrm{~mm}$ and $-270 \mathrm{~V}$ at $\mathrm{y}=+42 \mathrm{~mm}$ (black line). After one hour with $-40 \mathrm{kV}$, the maximal surface potential is approximately $-350 \mathrm{~V}$ (blue line). Applying $50 \mathrm{kV}$ for one hour results in maximal $-750 \mathrm{~V}$ (red line), after one hour $-55 \mathrm{kV}$, the maximal accumulated surface potential is $-1400 \mathrm{~V}$ (green line), and after one hour $-60 \mathrm{kV}-2400 \mathrm{~V}$ (magenta line). The surface potential exceeded the maximum measurement range after a further increase to $-65 \mathrm{kV}$ and application of 1 hour.

The simulated surface potential for the roughest electrode is compared to the measured one. Again only one half of the simulation results is shown in Figure $7 \mathrm{~b}$ ). An effective field enhancement factor of $\beta_{\text {eff }}=23$ (semi-ellipsoid with $I / r=6$ ) is necessary to reproduce the observed charge profiles. The prefactor $\mathrm{C}(\mathrm{V})$ increases for each voltage step up to $-60 \mathrm{kV}$ absolute voltage, indicating that an increasing fraction of the rough surface contributes to micro-discharges between voltages $(-20 \ldots-60 \mathrm{kV})$. 


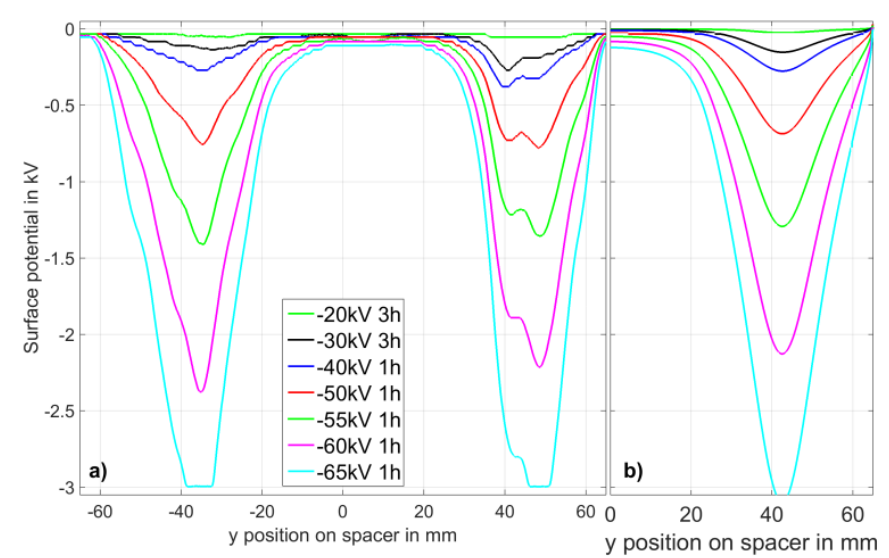

Figure 7 Surface Potential on the PTFE sample as a function of applied voltage and time for the $10.9 \mu \mathrm{m} \mathrm{R}_{\mathrm{a}}$ electrode. a) Measurement, b) Modeling.

\subsection{COATED ELECTRODE SURFACE}

Performing the experiment with a polished ring that was coated with $60 \mu \mathrm{m}$ paint did not lead to any measurable discharge up to a voltage of $-100 \mathrm{kV}(17.7 \mathrm{kV} / \mathrm{mm})$. Coating the F1000 ring with $60 \mu \mathrm{m}$ paint thickness did result in a surface roughness of $\mathrm{R}_{\mathrm{a}}=0.61 \pm 0.08 \mu \mathrm{m}$. Performing an experiment with an F1000 ring coated showed a significant improvement of the transition of the voltage current relationship due to micro discharges. Up to a voltage of $90 \mathrm{kV}$, only one spot at the whole sample showed additional charges. Increasing the voltage to $-100 \mathrm{kV}$ did result in a surface potential larger than the $-3 \mathrm{kV}$ value measurable with our probe, over the whole sample. This indicates a very strong micro discharge activity at this voltage level. No burned marks due to micro discharges could be detected with the naked eye at the coating after the experiment.

\section{Discussion}

The partial discharge measurement performed according to IEC 60270 did not show any partial discharges even for the roughest ring up to $-100 \mathrm{kV}$. However the measurements presented in Figure 6 and Figure 7 show substantial charging. These charges have to originate from discharges at the rough ring as the only other active ion source in these experiments is natural ionization, a process that was shown to increase the surface potential only by a few volts per hour in our experimental arrangement.

Thus, discharges have to take place that cannot be detected by classical PD measurement. These can be very small discharges with low amplitude or continuous discharges.

This observation is very important for designing and constructing HVDC GIS equipment in the future. It shows that even in a classical "PD-free" case, ion sources can be active that generate a large amount of charges in the gas phase. These charges can accumulate on the surface of insulators, distort the field distribution and may thereby influence (reduce) the withstand voltage of the equipment.

In section 4.1, it was shown that the polished ring does not generate additional charges. The surface potential increase in that case is in the range of $3 \mathrm{~V}$ per hour, a result consistent with natural ionization being the only charge source in the gas phase. Thus, it can be concluded that no micro discharge takes place in the case of the polished ring up to a maximal measured field strength of $17.7 \mathrm{kV} / \mathrm{mm}$ at $0.5 \mathrm{MPa} \mathrm{SF}_{6}$.

Figure 8 shows the simulated emitted current density on the electrode ( $r=42.5 \mathrm{~mm}$, point of maximum macroscopic field) after $1 \mathrm{~h}$ of voltage application on the PTFE sample as a function of negative applied voltage for the electrodes with $\mathrm{R}_{\mathrm{a}}=2.1$ and $\mathrm{R}_{\mathrm{a}}=10.9 \mu \mathrm{m}$. The micro-discharge current is determined by the local electric field at the electrode surface. As this local electric field strength decreases with increasing amount of charges accumulated on the PTFE sample in our arrangement, the emitted current decreases with time. The micro-discharge current shortly after voltage application is higher than the value displayed here. As an example, the maximum current density on the PTFE plate after $1 \mathrm{~h}$ at $-75 \mathrm{kV}$ on the F80 ring and pre-charging by lower voltages (-40 to -70 $\mathrm{kV}$ ) is $2.6 \times 10^{-9} \mathrm{~A} / \mathrm{m}^{2}$ (see Figure 8). If no pre-charging takes place (single step of $-75 \mathrm{kV}$ on F80 ring, uncharged PTFE), the modeled current density is about 20x higher after 10s voltage application $\left(5.7 \times 10^{-8} \mathrm{~A} / \mathrm{m}^{2}\right)$. This latter current might be more relevant for the application in real-size GIS, where the distance between the emitting surface and the insulator can be larger, and hence the screening effect by deposited charges lower.

The micro-discharge current for the F1000 ring increases gradually with increasing field strength, with no observable sharp transition. At the contrary, a steep transition occurs in the current-voltage characteristics of the F80 ring around 12 $\mathrm{kV} / \mathrm{mm}$ applied electric field. However, both electrodes induce more additional gas ions than natural ionization already from the lowest applied absolute voltages (see Figure 9 for comparison). This indicates that micro-discharges dominate at absolute voltages lower than the lowest measured voltages of $20 \mathrm{kV}$ and $40 \mathrm{kV}$ respectively.

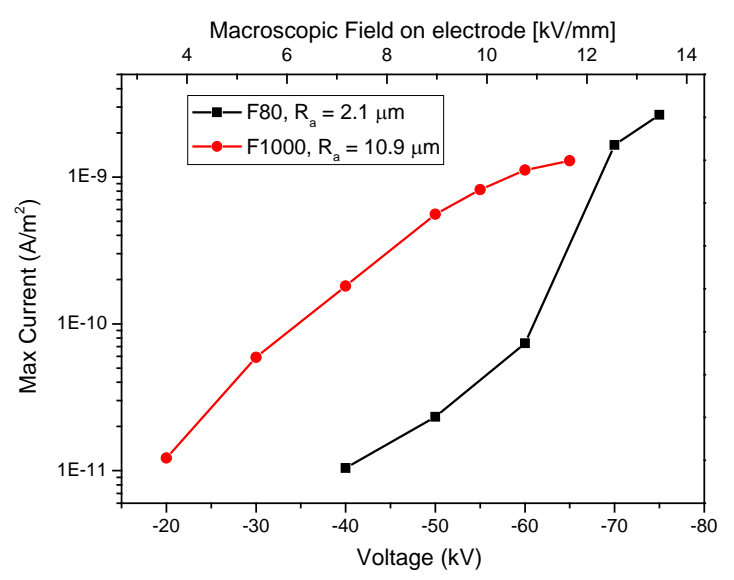

Figure 8 Left y-axis: Simulated current-voltage characteristics at the point of maximum macroscopic field $(r=42.5 \mathrm{~mm})$ corresponding to the measured surface potential increase $\Delta V$ during 1 h (Right $y$-axis). Current density value after $1 \mathrm{~h}$ of voltage application. As a comparison, current density from natural ionization corresponds to $2 \cdot 10^{-14} \mathrm{~A} / \mathrm{m}^{2}$ at this position.

Insulator surface charging from micro-discharges may dominate already at field strengths lower than previously 
expected, if bare metal electrodes with technical-grade roughness are present in DC GIS.

In Figure 5, the results obtained using a ring electrode with a roughness of $2.1 \mu \mathrm{m}$ are shown. This roughness value is the most interesting one as it is close to the roughness of real GIS applications.

The effect of the reduction of the macroscopic field due to accumulated surface charges can be seen in the measurements presented in Figure 5. At $-70 \mathrm{kV}$, the increase in surface potential from hour 1 to hour 2 is $419 \mathrm{~V}$, but from hour 2 to hour 3 the increase is only $9 \mathrm{~V}$. The accumulated charges have negative polarity. Thus the electric field at the ring is reduced due to the field caused by the accumulated charges. Due to the reduced field, the critical field $\mathrm{p}(\mathrm{E} / \mathrm{p})_{\text {crit }}$ at the micro protrusions is not exceeded anymore and the micro discharges stop. The same effect can be seen at $-65 \mathrm{kV}$. This saturation effect limits the total charge accumulated on an insulator surface. The effect of micro discharges at low fields in real HVDC components could be reduced by these accumulated charges for electrode surfaces close to the insulator like shown in [12]. For larger distances this effect is of limited influence as screening of the rough metal surface by accumulated charges on the insulator becomes less effective.

In Figure 7, results obtained using a ring with a roughness of $10.9 \mu \mathrm{m}$ are shown. Like explained in section 2.1.3, for large roughness $\mathrm{R}_{\mathrm{a}}$ many small and sharp micro protrusions are present but larger and blunter micro protrusions as well [14]. With an increase in the applied voltage, the slowly increasing ionic current observed in measurements can be attributed to the slowly increasing amount of micro protrusions causing discharges. This can also be seen in the current-voltage characteristics of Figure 8.

The current density impinging on the PTFE insulator is directly obtained from the simulation (see Figure 9b). Note that in this particular configuration, micro-discharges at -20 $\mathrm{kV}$ in front of the rough electrode (radius $=42.5 \mathrm{~mm}$ ) already exceed the maximum current from natural ionization. For increasing applied voltages, micro discharges are induced on a wider portion of the electrode, see Figure 9 insets c) and d).

The level of natural ionization sets the lower limit for detectable micro discharges, in this particular case $j=\dot{\rho}_{\mathrm{IP}} e \Delta z=2$. $10^{-14} \mathrm{~A} / \mathrm{m}^{2}$ [25] as seen in Figure 9. At the other extreme, the micro discharge regime ends when the streamer criterion is fulfilled [37]. The streamer inception threshold depends on local field enhancement at the surface, and hence on surface roughness. We calculated the streamer inception level by adding the field enhancement due to a single micro protrusion to the macroscopic field obtained from our finite element model. We used a semiellipsoid-on-a-post model, where the total length of the micro protrusion is chosen according to the measured surface roughness $\mathrm{R}_{\mathrm{t}}$ value (maximum height of the profile), and its aspect ratio was chosen to correspond to the $\beta_{\text {eff }}$ used in the model. As seen in Table 1 , the streamer inception voltage is always considerably higher than the observed onset voltage for micro discharges. The onset definition used here is the voltage value at which the current density caused by natural ionization is exceeded. This confirms that electron impact ionization is not active in most of the gas gap between electrode and PTFE plate, and the applicability of
Townsend's saturation current model used equation (4) is justified.

\begin{tabular}{|c|c|c|c|c|}
\hline $\begin{array}{l}\text { Sandblast } \\
\text { grain size }\end{array}$ & $\begin{array}{l}\text { Measured } \\
\text { roughness } \\
\mathbf{R}_{\mathbf{a}}[\mu \mathrm{m}]\end{array}$ & $\begin{array}{l}\text { Micro } \\
\text { discharge } \\
\text { onset } \\
\text { voltage } \\
\text { [kV] }\end{array}$ & $\begin{array}{l}\text { Onset } \\
\text { Field } \\
\text { [kV/mm] }\end{array}$ & $\begin{array}{l}\text { Streamer } \\
\text { inception } \\
\text { voltage } \\
{[\mathbf{k V}]}\end{array}$ \\
\hline Polished & 0.06 & $>100$ & $>17.7$ & 250 \\
\hline F80 & 2.1 & $<40$ & $<7.1^{*}$ & $>200$ \\
\hline F1000 & 10.9 & $<20$ & $<3.6$ & $>100$ \\
\hline $\begin{array}{l}\text { Polished } \\
\text { coated }\end{array}$ & $\begin{array}{l}\text { not } \\
\text { measured }\end{array}$ & $>100$ & $>17.7$ & - \\
\hline $\begin{array}{l}\text { F1000 } \\
\text { coated }\end{array}$ & 0.61 & 100 & 17.7 & - \\
\hline
\end{tabular}

The effect of coated electrodes was studied as well. Coating a polished ring did not show any effect in our measurements. Up to the maximal applied voltage of $-100 \mathrm{kV}$, no micro discharge could be observed.

Coating the roughest ring with a $60 \mu \mathrm{m}$ paint layer did result in a reduced measured roughness of $0.61 \pm 0.08 \mu \mathrm{m}$ and showed a significant reduction of the measured micro discharge activity, even though some metal tips of the ring did stick out of the paint. With the coating, the onset of micro discharges was raised to $-100 \mathrm{kV}$. This can be attributed to the partial covering of the sharp micro protrusions by the paint.

In Table 2, the results of our experiment series are summarized. The roughness of each ring and its micro discharge onset voltage and field strength is shown. Furthermore, the theoretical streamer inception voltage was calculated for the uncoated rings. In real AC GIS applications, the maximal electric field strength does normally not exceed $10 \mathrm{kV} / \mathrm{mm}$ [38]. This value might not be taken as design rule for HVDC gas insulated systems according to our findings. The roughness of $\mathrm{R}_{\mathrm{a}}=2.1 \mu \mathrm{m}$ represents real GIS electrode surfaces quite well. Like shown in Table 2, the discharge current due to micro discharges increases over the level of natural ionization at an electric field strength of $7.1 \mathrm{kV} / \mathrm{mm}$. Thus in a real GIS application insulator, charging from ions formed by micro discharges has to be expected. To avoid this charging in real DC GIS applications using the same materials as in AC GIS, the electrical field strength of $7.1 \mathrm{kV} / \mathrm{mm}$ should not be exceeded. Another possibility to avoid this unwanted charge source is to use smoother electrodes, see polished measurements shown in Table 2. Coating the electrode can also be of positive effect like shown with the F1000 coated measurement but as discussed before, a possible influence of the used paint has to be studied in detail. 

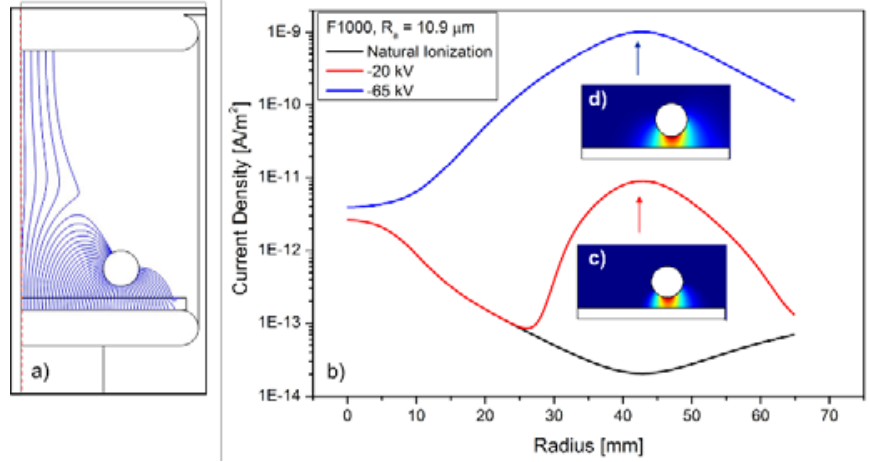

Figure 9 a) Field line in contact with the PTFE sample (active gas volume). b) Average current density on the PTFE plate sample for ions from natural ionization only (black line), and with additional ion generation from the electrode with $10.9 \mu \mathrm{m} \mathrm{R}$ at $-20 \mathrm{kV}$ and $-65 \mathrm{kV}$, respectively. Insets: c) and d) corresponding ion density in the gas originating from the rough electrode surface.

\section{ConcLUSION AND OUTLOOK}

In this paper we investigated the relation between surface roughness and currents originating from micro discharges for different electrode surface field strengths. The effect of micro discharges is quantified by measuring surface potential accumulation on PTFE plate samples. The current-voltage characteristics of each electrode roughness grade are then obtained by FEM modeling of the ion generation, driftdiffusion and surface accumulation processes.

In Table 2, a summary of the micro discharge onset voltage (and field) as function of surface roughness is presented. An important finding is that micro-discharges may dominate gas conduction already at much lower field strength than previously expected when electrode surfaces with technicalgrade roughness are present in the system. However, limited charge accumulation on neighboring insulating surfaces is sufficient to reduce the field to sub-critical values and stop the micro discharge activity after a few hours of DC stress slightly above the onset voltage.

Current-voltage characteristics for certain surface roughness grades are given which may serve as a base for design rules to avoid micro discharges when constructing gas insulated HVDC equipment.

Significant increase of the micro discharge inception voltage is demonstrated when coating a rough electrode with a slightly conductive paint. However, further measurements have to be done to assess long term behavior of such paints and to draw conclusions on their general applicability on HVDC components.

Finally, the chosen modeling approach of ion generation and drift in the gas delivers amplitude and spatial distribution of the ionic current density impinging on insulators. Therefore, these results can be transferred to real size GIS components for determining design field strengths on technical surfaces, thus controlling micro discharges' occurrence and subsequent detrimental charge accumulation on insulator surfaces.

\section{ACKNOWLEDGMENT}

RG and CBD thank Sergey Pancheshnyi for inspiring discussions.

\section{REFERENCES}

[1] T.J. Hammons, V.F. Lescale, K. Uecker, M. Haeusler, D. Retzmann, K. Staschus and S. Lepy, "State of the Art in Ultrahigh-Voltage Transmission”, Proc. IEEE, Vol. 100, No. 2, pp. 360-390, 2012.

[2] M.P Bahrman and B.K Johnson, "The ABCs of HVDC transmission technologies”, IEEE Power Energy Mag., Vol. 5, No. 2, pp. 32-44, 2007.

[3] C. W. Mangelsdorf and C. M. Cooke, "Static Charge Accumulated by Epoxy Post Insulation Stressed at High DC Voltages", IEEE Conf. Electr. Insul. Dielectr. Phenomena (CEIDP), pp. 220-227, 1978.

[4] S.I. Bektas, O. Farish and M. Hizal, "Computation of the electric field at a solid/gas interface in the presence of surface and volume charges", IEE Proc-A, Vol. 133, No. 9, pp. 577-586, 1986.

[5] S.M. Gubanski, "Role of surface charging in the performance of polymeric high voltage insulation systems”, IEEE Int'l. Conf. Solid Dielectrics (ICSD), pp. 1-11, 2010.

[6] A. Winter, Elektrische Ladungen auf Isolierstoffoberflächen und deren Wirkung auf die Durchschlagsspannung von Elektrodenanordnungen, Ph.D. thesis, Technical University Munich TUM, Germany, 2003.

[7] T. Jing, "Surface Charge Accumulation in SF6, Mechanism and Effects, Ph.D. thesis, Delft University of technology, The Netherlands, 1993.

[8] F. Messerer, Gas-Insulated Substation (GIS) for HVDC, PhD thesis, Technical University Munich TUM, Germany, 2001.

[9] B. Lutz, J. Kindersberger, "Surface Charge Accumulation on cylindrical polymeric model Insulators in air: Simulation and Measurement”, Trans. Dielectr. Electr. Insul., Vol. 18, No. 6, pp. 2040-2048, 2011.

[10] M. Schueller, U. Straumann and C. M. Franck, "Role of ion sources for Insulator Charging in SF6 Gas Insulated HVDC Systems”, IEEE Trans. Dielectr. Electr. Insul., Vol. 21, Issue 1, pp. 352-359, February 2014.

[11] C.M. Cooke, "Charging of insulator surfaces by ionization and transport in gases”, IEEE Trans. Dielectr. Electr. Insul., Vol. 17, No. 2, pp. 172178, 1982.

[12] K. Nakanishi, A. Yoshioka, Y. Arahata, Y. Shibuya, "Surface Charging on Epoxy Insulator at Dc stress in compressed SF6 gas", IEEE Trans. Power App. Syst. Vol.PAS-102, No.12, pp. 3919-3927, 1983.

[13] H. Fujinami, T. Takuma, M. Yashima, T. Kawamoto, "Mechanism and effect of DC charge accumulation on $\mathrm{SF}_{6}$ gas insulated insulators", IEEE

Trans. on Power deliv., Vol.4, No.3, pp. 1765-1772, 1989.

[14] P.W. Karlsson, A. Pedersen, "Inherent Limitations in Uniform Field Discharge Data for SF6”, IEEE Trans. Power App. and Systems, Vol.91, No.4, pp. 1597-1601, 1972.

[15] S. Berger, "Onset or breakdown voltage reduction by electrode surface roughness in air and SF6", IEEE Trans. Power App. and Systems, Vol 95, No. 3, 1073-1079, 1976.

[16] S.M. El-Makkawy, "Electrode surface roughness initiated breakdown in compressed SF6 gas", IEEE Conf. Electr. Insul. Dielectr. Phenomena (CEIDP), pp 948-953, 1994.

[17] Y. Inagawa, K. Kato, et.al., "Effect of electrode surface roughness on breakdown conditioning process under non-uniform electric field in vacuum", Symp. on Discharges and Electrical Insulation in Vacuum, pp. 72-75, 2004.

[18] O. Farish, O.E. Ibrahim, B.H. Crichton, "Effect of electrode surface roughness on breakdown in nitrogen/SF6 mixtures", Proc. of Electrical Engineers, Vol. 123, No. 10, pp. 1047-1050, 1976.

[19] N. Wiegart, L. Niemeyer, F. Pinnekamp, W. Boeck, J. Kindersberger, R. Morrow, W. Zaengl, M. Zwicky, I. Gallimberti, and S.A. Boggs. "Inhomogeneous Field Breakdown in GIs-Part II: Ion density and Statistical Time Lag”, IEEE Trans. on Power Delivery, Vol 3, No. 3, July, p. 931, 1988.

[20] X.M. Bian, L. Chen, et al. "Surface roughness effects on the corona discharge intensity of long-term operating conductors." Applied Physics Letters 101(17), 2012. 
[21] S. Boggs, R. J. Densley, "Fundamentals of partial discharge in the context of field cable testing" IEEE Electrical Insulation Magazine", Vol. 16, No. 5, pp. 13-18, Sept.-Oct. 2000.

[22] M. Saltzer, U. Gäfvert, et al. "Observation of space charge dynamics in air under DC electric fields" CEIDP, Cancun, Mexico, 2011.

[23] U. Straumann, M. Schueller, C.M. Franck "Theoretical Investigation of HVDC Disc Insulator Charging in SF6 Gas Insulated Systems”, IEEE Trans. Dielectr. Electr. Insul., Vol. 19, No. 6, pp. 2196-2205, 2012.

[24] T. Christen, The Boundary Conditions for Field Simulations in Insulators. 4. ETG-Fachtagung Grenzflächen in elektrischen Isoliersystemen. Dresden, Germany, 2013.

[25] R. Gremaud, F. Molitor, et al. "Solid-gas interfaces in DC gas insulated systems”, 4. ETG-Fachtagung Grenzflächen in elektrischen Isoliersystemen, Dresden, Germany, 2013.

[26] J.S. Townsend, (1914). Philos. Mag. 28: 83.

[27] R.S. Sigmond, "Simple approximate treatment of unipolar spacecharge-dominated coronas: The Warburg law and the saturation current." Journal of Applied Physics 53(2): 891-898, 1982.

[28] Q.J. Feng, "An analysis of corona currents between two concentric cylindrical electrodes." Journal of Electrostatics 46(1): 37-48, 1999.

[29] J.S. Townsend, (1914). Philos. Mag. 28: 83.J.S.

[30] R.S. Sigmond, "Simple approximate treatment of unipolar spacecharge-dominated coronas: The Warburg law and the saturation current." Journal of Applied Physics 53(2): 891-898, 1982.

[31] I.W. McAllister, "Electric fields and electrical insulation." Dielectrics and Electrical Insulation, IEEE Transactions on 9(5): 672-696, 2002.

[32] A. Bendaoud, A. Tilmatine, et.at.: "Experimental Study of Corona Discharge Generated in a Modified Wire Plate Electrode Configuration for Electrostatic Processes Applications” IEEE IAS 08, Edmonton, Canada, 2008.

[33] R.G. Forbes, C.J. Edgcombe, et al. "Some comments on models for field enhancement." Ultramicroscopy 95(0): 57-65, 2003.

[34] M. Schueller, U. Straumann and C. M. Franck, "Time dependent insulator charging in SF6 under DC stress", IEEE Conf. Electr. Insul. Dielectr. Phenomena CEIDP, pp. 629-632, Montreal, Canada, 2012.

[35] DuPont, Teflon PTFE, Properties Handbook, retrieved online at http://www.rjchase.com/ptfe handbook.pdf on January 2015.

[36] M. Schueller, C.M. Franck, "Influence of the gas volume size on insulator charging in SF6 under DC stress", Proc. XVIII ISH, Seoul, South Korea, 2013.

[37] L. Niemeyer, "A Generalized Approach to Partial Discharge Modeling" IEEE Trans. Diel. Electrical Insulation 2: 510, 1995.

[38] E. Volpov, "Dielectric strength coordination and generalized insulator design rules for HVAC/DC SF6 gas insulated systems" IEEE Trans. Dielectr. Electr. Insul., Vol. 11, Issue 6, pp. 949-963, Dec. 2004.

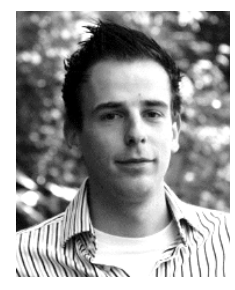

Michael Schueller (M'12) received a diploma in Electrical Engineering with honors from the Faculty of Electrical and Information Engineering from TU Graz, Austria in 2009. Currently he is doing his Ph.D. at the Institute for Power Systems and High Voltage Technology, ETH Zürich, Switzerland. His research interests cover gas insulated High Voltage Systems for DC applications and charge accumulation associated with High Voltage equipment under DC stress.

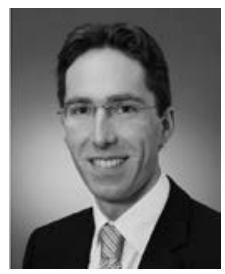

Robin Gremaud received a diploma in physics from the University of Fribourg, Switzerland, in 2003, and his Ph.D. degree in physics from the VU University Amsterdam, Netherlands, in 2008. After a postdoc position 2008-2010 at the research institute for materials science EMPA, Switzerland, he joined the Swiss ABB corporate research center in 2010 and is currently Senior Scientist in the Applied Physics group. His current research addresses the physics of solid and gas insulation under HVDC stress.

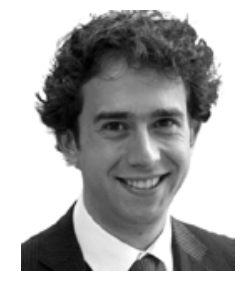

Charles B. Doiron received his B.Sc and his M.Sc. degrees in physics from the University of Sherbrooke, Canada, in 2002 and 2005, respectively, and his Ph.D. degree in theoretical physics from the University of Basel, Switzerland, in 2009. He joined the Swiss corporate research center of ABB in 2009, where he currently works as Senior Scientist in the Theoretical Physics group, on topics related to thermodynamic and dielectric properties of gases.

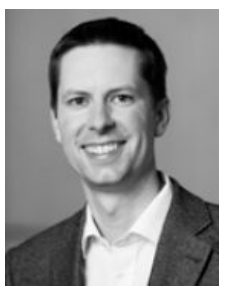

Christian M. Franck (M'04-SM'11) received a diploma in physics from the University of Kiel, Germany in 1999 and the Ph.D. degree in physics from the University of Greifswald, Germany in 2003. He was with the Swiss corporate research center of ABB from 2003-2009 as a Scientist and Group Leader for gas circuit breakers and high-voltage systems. Currently, he is Assistant Professor for High Voltage Technology at the Swiss Federal Institute of Technology (ETH), Zürich, Switzerland. 